\title{
«Pas s'énerver, juste s'étonner»
}

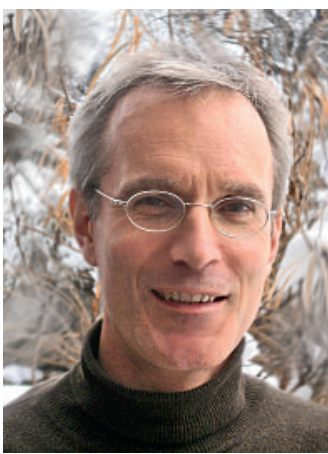

Bruno Kesseli
C'est ce conseil minimaliste que le président de la FMH, Jacques de Haller, m'a adressé par voie électronique au cours de l'année qui touche à sa fin. Dans le cadre d'une discussion interne à la rédaction menée par le biais d'e-mail, j'avais précédemment qualifié d'«énervant» un passage dans une contribution. J'ai depuis oublié la raison de mon mécontentement, ce qui laisse à penser que le conseil donné par mon collègue était avisé. Quoi qu'il en soit, le bon mot de notre président n'a depuis eu de cesse de trotter dans ma tête. Il faut dire que les occasions ne manquent pas au quotidien.

Parmi les points positifs, il est à noter que le travail de rédacteur en chef du BMS donne régulièrement l'occasion de cultiver la vertu de l'impassibilité. Les idées quant à l'orientation du BMS en termes de forme et de contenu et aux intérêts qu'il est censé défendre semblent en effet se polariser en ces temps mouvementés pour la politique (de santé). D'aucuns souhaitent apparemment que le BMS soit le fer de lance contre les tendances néolibérales dans le domaine de la santé alors que d'autres s'émeuvent à cause d'une caricature de Blocher de l'instrumentali-

\section{Nous notons cependant avec satisfaction que le BMS est lu avec assiduité et jugé important pour l'exercice de la profession.}

* REMP Recherches et études des média publicitaires est un organe central et neutre qui a pour mission d'instaurer la transparence sur le marché des médias et de la publicité. La reconnaissance d'études de marché par REMP est soumise à des critères très rigoureux qui sont scrupuleusement vérifiés.

** Les principaux résultats de l'étude peuvent être consultés sous www.gfs-zh.ch $\rightarrow$ Leserumfrage medizinische Fachpresse. Des analyses complètes peuvent être commandées contre paiement sur www.gfs-zh.ch. sation politique déplacée du BMS à des fins de gauche. D'autres encore peinent à accepter les règles et les compétences de la rédaction définies dans les Directives pour les auteurs. Une collègue souhaite davantage de contributions culturelles, car la culture fait précisément les frais du quotidien professionnel, un autre voudrait que soit privilégié le rapport à l'actualité du jour, etc. Nous ne pouvons que répéter ce qui a déjà été souligné en de précédentes occasions: le BMS ne peut pas faire siens des intérêts particuliers. Il a en effet pour mission de répliquer le paysage de la politique de santé et notamment les positions du corps médical dans toute sa diversité.

Je ne voudrais pas donner l'impression de me plaindre et tiens donc à souligner qu'après cinq ans, mon travail pour le BMS me plaît plus que jamais. Cela s'explique par le bon climat de travail chez EMH et par l'équipe de rédaction engagée que je tiens à remercier ici pour son travail, mais aussi en grande partie par vous, chers collègues. Car ainsi que j'en fais le constat quasi quotidien, notre lectorat est certes critique et ne s'embarrasse pas toujours de circonvolutions, mais il sait aussi se montrer très constructif dans les contacts directs et apprécie nos efforts pour fournir un service de qualité. Il est toujours stimulant de s'en rendre compte.

Nous avons récemment reçu d'un organisme au-dessus de tout soupçon la confirmation, en cette période de fête, de nos impressions subjectives concernant l'importance du BMS pour ses lecteurs. Une enquête à grande échelle de l'Institut für Markt- und Sozialforschung gfs-zürich qui a obtenu le label convoité REMP* a confirmé que le Bulletin des médecins suisses se place loin devant la concurrence en termes de portée nationale. Les autres magazines édités par les Editions médicales suisses EMH, tels que le Forum médical suisse (FMS), PrimaryCare ou le Swiss Medical Weekly (SMW) ont d'ailleurs eux aussi obtenu d'excellents résultats**.

Il est vrai que l'étude a été commandée par les éditions Groupe Médecine et Hygiène et les Editions médicales suisses EMH (et soutenue par la FMH, Interpharma et vips). Mais des instituts renommés tels que gfs-zürich, sans même parler de REMP, se garderont bien de produire des résultats de complaisance, car les conséquences sur leur réputation seraient désastreuses. Avec plus de 2800 médecins ayant participé à l'enquête, cette étude atteint également un niveau jusqu'à présent inégalé pour ce qui est des données exploitables.

La position de tête incontestée du BMS en termes de diffusion n'a évidemment pas de quoi surprendre. Nous sommes parfaitement conscients du monopole dont nous bénéficions d'une certaine manière en tant qu'organe officiel de la FMH. Nous notons cependant avec satisfaction que le BMS est lu avec assiduité et jugé important pour l'exercice de la profession. Des valeurs de pointe sont obtenues même dans les dimensions qualitatives, le lectorat de Suisse alémanique nous conférant des notes un peu meilleures que les collègues de Suisse romande (nous pouvons y féliciter la Revue Médicale Suisse pour ses excellents résultats).

Nous n'allons pas nous reposer sur nos lauriers, mais l'encouragement exprimé par l'enquête auprès de nos lecteurs est le bienvenu pour débuter la nouvelle année. Au nom de la rédaction, je vous souhaite de joyeuses fêtes et vous adresse tous mes vœux pour 2011.

Bruno Kesseli, rédacteur en chef 